\title{
Vigilancia del uso de medicamentos en el embarazo en el municipio de Los Palmitos, Sucre, Colombia: una contribución para la prevención y reducción de la mortalidad materna y perinatal
}

\author{
Surveillance of drug use in pregnancy in the municipality of Los Palmitos, \\ Sucre, Colombia: a contribution for the prevention and reduction of \\ maternal and perinatal mortality
}

Marco Antonio Márquez Gómez ; Graciela María Gómez Díaz ${ }^{2}$

\section{Resumen}

Objetivo. Evaluar una estrategia de vigilancia del uso de medicamentos en embarazadas en un municipio colombiano. Métodos. Estudio de utilización de medicamentos, tipo descriptivo, corte transversal, recolección prospectiva de la información, encaminado a identificar los medicamentos consumidos por embarazadas sin prescripción médica. Mediante muestreo aleatorio simple con IC 95\% y EM 5\%, se obtuvo una muestra de 155 maternas. Resultados. Se diseñó, validó y aplicó un instrumento de recolección de información, aplicado a 169 embarazadas, de las cuales 152 cumplieron cuatro controles de embarazo. La edad promedio fue 24.5 años (Min. 13 y Max. 40), viven en unión libre, y solo tienen estudios secundarios. El 42\% tenía su primer embarazo, y el $30 \%$ ha tenido 3 o más embarazos. El promedio de hijos fue de 1.0, pero 13 de ellas tenían más de 3 hijos. Las embarazadas asistieron a 4 controles en promedio. El índice de automedicación fue de $21 \%$. Las embarazadas consumieron 11 productos sin prescripción médica; 9 medicamentos de síntesis, y 2 plantas medicinales. La frecuencia de consumo de productos clasificados con riesgo B fue del $29 \%$, riesgo C fue del $33 \%$, y para las categorías D y X fue del $38 \%$. Se identificaron dos plantas medicinales (Equisetum giganteum L., Pedilanthus tithymaloides L. Poit) con potencial riesgo para el embarazo. Conclusiones. El instrumento ayuda a la identificación de características cuyo control puede contribuir a la maternidad segura, y permite evidenciar el uso de productos con potencial riesgo para el embarazo.

Palabras claves: Embarazo, Fitoterapia, Estudios de seguimiento, Medicamentos, Mortalidad materna, Mujeres embarazadas, Riesgo, Utilización de Medicamentos, Vigilancia.

\section{Abstract}

Objective. To evaluate a strategy to monitor the use of drugs in pregnant women in a Colombian municipality. Methods. Drug use study, descriptive type, cross-sectional, prospective collection of information, aimed at identifying medications consumed by pregnant women without a medical prescription. Using a simple random sample with 95\% CI and 5\% MS, a sample of 155 maternal

1. Químico Farmacéutico, Docente Escuela de Ciencias de la Salud, Universidad Nacional Abierta y a Distancia. Grupo de investigación

TECNNOSALUD. Colombia.

2. Tecnóloga en regencia de farmacia, Universidad Nacional Abierta y a Distancia. Grupo de investigación TECNNOSALUD. Colombia. 
samples was obtained. Results. A data collection instrument was designed, validated and applied to 169 pregnant women, of whom 152 met four pregnancy controls. The average age was 24.5 years (Min. 13 and Max. 40), live in free union, and only have secondary studies. $42 \%$ had their first pregnancy, and $30 \%$ had 3 or more pregnancies. The average number of children was 1.0 , but 13 of them had more than 3 children. Pregnant women attended 4 controls on average. The selfmedication rate was $21 \%$. The pregnant women consumed 11 products without prescription; 9 synthetic medicines, and 2 medicinal plants. The frequency of consumption of products classified as risk $\mathrm{B}$ was $29 \%$, risk $\mathrm{C}$ was $33 \%$, and for categories $\mathrm{D}$ and $\mathrm{X}$ was $38 \%$. Two medicinal plants (Equisetum giganteum L., Pedilanthus tithymaloides L. Poit) with potential risk for pregnancy were identified. Conclusions. The instrument helps to identify characteristics whose control can contribute to safe motherhood, and makes it possible to highlight the use of products with a potential risk for pregnancy.

Keywords: Pregnancy, Phytotherapy, Follow-Up Studies, Drugs, Maternal Mortality, Pregnant Women, Risk, Drug Utilization, Surveillance.

\section{Introducción}

A pesar de lograr avances en la salud materna, las complicaciones relacionadas con la gestación siguen siendo un importante problema de salud pública en el mundo. Cada año mueren $585.000 \mathrm{mu}$ jeres durante la gestación, el parto o el puerperio. Se presentan cerca de 50 millones de problemas en salud materna anualmente y aproximadamente 300 millones de mujeres sufren, a corto y largo plazo, de enfermedades y lesiones relacionadas con el embarazo, el parto y el puerperio (1).

La gestación es un periodo único durante el cual la exposición a un determinado fármaco involucra a dos organismos: la madre y el feto. Este último, mucho más sensible a sus efectos y toxicidad, puede sufrir graves problemas, incluso irreversibles. Entre los profesionales de la salud, el manejo de medicamentos en el embarazo representa siempre un punto crítico en la atención. La falta de conocimientos sobre cómo interpretar las evidencias sobre este tema, asociada a sesgos de observación de los profesionales, crea algunas situaciones en que la evaluación del riesgo se vuelve dudosa (2).
Durante el embarazo la mayoría de las mujeres consumen medicamentos, algunos estudios señalan que el $97 \%$ de las mujeres embarazadas consumen por lo menos un medicamento (3).

En el Departamento de Sucre las complicaciones del embarazo, parto y puerperio constituyen las principales causas de muerte de mujeres en edad reproductiva. La razón de mortalidad materna y mortalidad neonatal por 100.000 nacidos vivos, se calculó en el año 2011 en 84 y 5.1 respectivamente (4).

En la farmacia, principalmente en la ambulatoria, el papel del farmacéutico en el consejo a las embarazadas puede ser decisivo en la utilización o no de un medicamento exento de prescripción o incuso en la detección de medicamentos prescritos de alto riesgo teratogénico conocido (2). La situación descrita plantea unos importantes retos de investigación para el servicio farmacéutico debido a su condición de servicio de apoyo terapéutico; y la de sus integrantes como miembros del equipo de salud, agentes educadores y promotores de estilos de vida saludables. 


\section{Materiales y métodos}

\section{Tipo de estudio}

Con el objetivo de contribuir a la prevención y contención de la mortalidad materna y perinatal en mujeres embarazadas del municipio de Los Palmitos - Sucre, mediante la identificación de los medicamentos y plantas medicinales consumidas sin prescripción médica durante el embarazo, se diseñó un modelo de vigilancia farmacológica a partir de la metodología de los estudios de utilización de medicamentos y del seguimiento farmacoterapéutico para monitorear el consumo de medicamentos y productos naturales por automedicación; el estudio fue de tipo descriptivo, de corte transversal, con recolección prospectiva de la información.

\section{Población y muestra}

Para el cálculo de la muestra se tomaron dos datos de referencia:

1. Según las proyecciones poblacionales registradas por el DANE a partir de los datos obtenidos en el Censo 2005 (5), el número de mujeres en edad fértil, es decir de $10 \mathrm{a} 49$ años del municipio de Los Palmitos para el ańo 2014 era de 5.633 mujeres.

2. De acuerdo con la encuesta nacional de demografía y salud del año 2010, elaborada por Profamilia (6), en promedio en el departamento de Sucre el $4.6 \%$ de las mujeres en edad fértil se encuentran embarazadas en un momento dado.

Se asumió este factor departamental y se calculó una población de 259 mujeres embarazadas en el municipio de Los Palmitos; Luego se aplicó un muestreo aleatorio simple, con un $95 \%$ de confianza y un $5 \%$ de error, estimándose el tamaño de la muestra en 155 mujeres embarazadas.

La vigilancia del consumo de medicamentos y productos naturales sin prescripción médica se reali- zó durante cuatro meses en el centro de salud del municipio de Los Palmitos en el Departamento de Sucre. El seguimiento se realizó a la salida de cada control prenatal mediante una entrevista con las gestantes que decidieron participar en el estudio; se propuso un número mínimo de 4 entrevistas por materna.

\section{Variables de estudio}

Se analizaron las siguientes variables:

- Información sociodemográfica de las gestantes. Edad, procedencia, afiliación a seguridad social, estado civil, escolaridad.

- Información gineco-obstetrica. Número de partos, número de cesáreas, número de abortos, número de hijos nacidos vivos, semana del embarazo actual, número de controles prenatales a los que ha asistido.

- Índice de automedicación. Porcentaje de embarazadas que han consumido al menos un medicamento sin prescripción médica durante el embarazo.

- Riesgo en el embarazo de los productos consumidos. Clasificación del riesgo en el embarazo del medicamento o producto natural consumido según la guía de la FDA.

- Productos consumidos por automedicación. Frecuencia de consumo de los medicamentos reportados.

\section{Técnicas de recolección y análisis de la informa- ción}

Para la realización del estudio, se usó la encuesta como técnica de recolección de la información, para lo cual se diseñó un instrumento de recolección de información que involucró variables sociodemográficas, variables gineco-obstétricas y variables relacionadas con el consumo de medicamentos y productos fitoterapéuticos.

Se realizó un proceso de validación simple del instrumento de encuesta aplicándolo al $10 \%$ de la 
muestra calculada (16 pacientes) encontrándose que no ofrecía dificultad para su diligenciamiento y captura de información.

Los datos recolectados se procesaron en una hoja de cálculo de Microsoft Excel y se analizaron usando medidas de tendencia central y distribución de frecuencias.

\section{Aspectos éticos}

Teniendo en cuenta que los métodos, técnicas y estrategias propuestos para la realización del estudio no involucraron en ninguna fase la intervención o modificación intencionada de variables biológicas, fisiológicas o sociales de los individuos bajo estudio, o la identificación de aspectos sensitivos de su conducta, esta investigación se clasificó en la categoría de investigación sin riesgo, de conformidad con el artículo 11 de la resolución 8430 de 1993.

A cada materna se le informó sobre la realización del estudio, sus alcances e implicaciones y se le solicitó que decidiera si accedía a participar voluntariamente en el estudio. Todas las maternas entrevistadas accedieron libre y voluntariamente a participar.

\section{Resultados}

\section{Cumplimiento del número mínimo de entrevistas y reajuste de la muestra}

La encuesta fue aplicada durante un periodo de cuatro meses a las mujeres embarazadas que acudían al centro de salud, iniciándose el seguimiento a una cohorte de 169 maternas, sin embargo, al final del periodo se encontró que solo 152 habían alcanzado el número mínimo de 4 entrevistas propuestas por el equipo investigador. La muestra definitiva se reajusto en 152 maternas.

\section{Información sociodemográfica de las gestantes}

Todas las gestantes encuestadas eran amas de casa o no ejercían labor alguna al momento de la encuesta, todas estaban afiliadas al sistema de seguridad social en salud, en el régimen subsidiado.

La mayoría (68\%) residían en el casco urbano del municipio, mientras que el resto $(32 \%)$ provenían de la zona rural.

En cuanto al estado civil, el $87 \%$ de las encuestadas tenía algún tipo de unión estable con su pareja, mientras que el $13 \%$ restante manifestaron estar solteras o separadas.

Se encontraron 16 menores de edad entre las gestantes; de ellas, una con 13 ańos (menor edad registrada) y tres de 14 años que quedaron embarazadas antes de cumplirlos. Por otra parte, 15 gestantes eran mayores de 34 ańos, particularmente se encontró un caso de una materna con 40 años (máxima edad registrada). Ver Tabla 1.

Tabla 1.Edad de las gestantes del municipio de Los Palmitos 2014. Fuente. Construcción de los autores.

\begin{tabular}{c|c|c}
$\begin{array}{c}\text { RANGO DE } \\
\text { EDAD }\end{array}$ & CANTIDAD & PORCENTAJE \\
\hline 10 a 17 & 16 & 11 \\
\hline 18 a 25 & 81 & 53 \\
\hline 26 a 33 & 40 & 26 \\
\hline 34 a 45 & 15 & 10 \\
\hline TOTAL & 152 & 100 \\
\hline
\end{tabular}

El promedio de edad de las gestantes fue de $25 \pm 6$ años.

La escolaridad de la población en general es baja, solo un $4 \%$ ha alcanzado estudios universitarios, el $88 \%$ solo terminó la secundaria, y un $8 \%$ de la población de gestantes no tiene ningún grado de formación académica.

\section{Información gineco-obstetrica}

En su mayoría (42\%) las mujeres eran primigestantes; $48 \%$ presentaban entre dos y cuatro embarazos 
incluyendo el actual; y el 10\% restante presentaba 5 o más embarazos.

El 26\% de las gestantes estaban en el primer trimestre del embarazo, el $35 \%$ en el segundo trimestre, y el $39 \%$ en el tercer trimestre.

El promedio de controles prenatales fue de 4 (Min: 1 y Max: 9), en las maternas que estaban en tercer trimestre del embarazo se encontró que 26 de ellas tenían asistencia a menos controles que los teóricos para este periodo (mínimo 6, máximo 9 controles); en el grupo que estaba en segundo trimestre la inasistencia fue de solo 6 casos.

Cada materna había tenido en promedio 0.8 partos previos, 0.2 cesáreas, 0.3 abortos y 0.9 hijos nacidos vivos. La relación parto/cesárea fue de 4:1; los partos representaron el $80 \%$ de los nacimientos. Por cada 3 hijos nacidos vivos, en promedio las maternas habían tenido un aborto.

\section{Índice de automedicación}

32 gestantes de 152 encuestadas admitieron haber consumido medicamentos o productos fitoterapéu- ticos sin indicación médica en algún momento de su embarazo, calculándose en $21 \%$ la automedicación en gestantes del municipio de Los Palmitos Sucre. Aproximadamente 1 de cada 5 gestantes se auto-medicaron.

\section{Productos consumidos por automedicación}

Se identificaron 11 productos farmacéuticos que fueron consumidos por 32 gestantes en 52 oportunidades; 9 medicamentos de síntesis y 2 productos fito terapéuticos, los cuales fueron consumidos sin prescripción médica.

\section{Riesgo en el embarazo de los productos consumidos}

Para la determinación del riesgo de exposición a medicamentos se utilizó la guía rápida de consulta sobre las categorías de clasificación del riesgo de la FDA, usada por Abad, et al. (7), la cual clasifica los medicamentos en función del riesgo para el feto, dichas categorías de la menos riesgosa o más segura a la menos segura o contraindicada, comprende cinco asignaciones de letras que son: $\mathrm{A}, \mathrm{B}, \mathrm{C}, \mathrm{D}, \mathrm{X}$. Ver Tabla 2.

Tabla 2. Medicamentos y su clasificación de riesgo consumidos por las gestantes del municipio de Los Palmitos 2014. Fuente. Construcción de los autores.

\begin{tabular}{l|r|r}
$\begin{array}{c}\text { MEDICAMENTO O PRODUCTO FITO } \\
\text { TERAPÉUTICO CONSUMIDO }\end{array}$ & $\begin{array}{c}\text { FRECUENCIA } \\
\text { CONSUMO }\end{array}$ & $\begin{array}{c}\text { CLASIFICACIÓN DEL } \\
\text { RIESGO }\end{array}$ \\
Cola de caballo (Equisetum giganteum L.) & 15 & $\mathrm{D}$ \\
\hline Ibuprofeno & 9 & $\mathrm{C}$ \\
\hline Acetaminofén & 8 & $\mathrm{~B}$ \\
\hline Ampicilina & 5 & $\mathrm{~B}$ \\
\hline Itamo real (Pedilanthus tithymaloides L. Poit) & 5 & $\mathrm{X}$ \\
\hline Omeprazol & 3 & $\mathrm{C}$ \\
\hline Amoxicilina + Clotrimazol & 2 & $\mathrm{~B}$ \\
\hline Diclofenaco & 2 & $\mathrm{C}$ \\
\hline Ácido Acetilsalićlico & 1 & $\mathrm{C}$ \\
\hline Gentamicina & 1 & $\mathrm{C}$ \\
\hline Metocarbamol & 1 & $\mathrm{C}$ \\
\hline
\end{tabular}


La frecuencia de consumo de productos clasificados con riesgo B fue del 29\%, con riesgo C fue del $33 \%$, y para las categorías D y X fue del $38 \%$; estas últimas correspondientes a dos plantas medicinales con alto riesgo para el embarazo $(8,9)$.

\section{Discusión}

\section{Utilidad del instrumento}

El instrumento fue aplicado a 152 maternas en al menos 4 oportunidades correspondientes a los 4 controles mínimos propuestos por el equipo investigador, lo que garantizó el seguimiento a todas las gestantes que participaron, en por lo menos cuatro controles; la vigilancia farmacológica realizada mediante la aplicación del instrumento durante las entrevistas permitió identificar el consumo de plantas medicinales con potencial riesgo para el feto.

\section{Condiciones socio-demográficas}

Las condiciones socio-demográficas de las gestantes del municipio de Los Palmitos reflejan los problemas propios de un municipio con un alto índice de necesidades básicas insatisfechas y son determinantes de las principales condiciones de salud que ponen en riesgo la maternidad; en efecto, el municipio presenta un $54.68 \%$ de necesidades básicas insatisfechas reflejada en una frágil infraestructura sanitaria (baja cobertura de suministro de agua potable, recolección de residuos sólidos, alcantarillado, acceso a la salud, etc.) y que se refleja en una alta tasa de mortalidad en menores de un año $(8.8$ x 100.000 habitantes), alta tasa de mortalidad en menores de 5 años (170 x 100.00 habitantes), alto porcentaje de nacidos con bajo peso $(9.89 \%)$, alta tasa de violencia intrafamiliar (77.7 casos x 100.000 habitantes), entre otras variables de alto impacto en la población $(4,12)$.

\section{Edad de las gestantes}

Si bien el 90\% de las encuestadas es menor de 33 años lo que revela la juventud de la población ges- tante, se han encontrado casos preocupantes en términos de riesgo para la madre y el feto tanto por maternidad temprana como tardía.

\section{Maternidad temprana}

El embarazo adolescente es un tema de reciente preocupación en la sociedad y para las autoridades de salud constituyendo un problema social y médico, por sus implicaciones en la condición física, mental y económica de la embarazada, y por el riesgo para el desarrollo del feto (4, 10-12).

Las mujeres jóvenes tienen mayores dificultades para el control de la fecundidad, debido a múltiples factores de riesgo. En el municipio de Los Palmitos esta situación adquiere características importantes si se tiene en cuenta la presencia de estos factores de riesgo en el municipio tales como la procedencia urbana o rural y el nivel de escolaridad (12).

En la cohorte estudiada el porcentaje de embarazo adolescente fue de solo el $10.5 \%$, muy por debajo del $23.8 \%$, porcentaje registrado en el análisis de situación de salud del municipio (12), y del 24.8\%, reportado para el departamento de Sucre (4).

\section{Maternidad tardía}

La determinación de la edad óptima para un embarazo ha sido un asunto de controversia entre profesionales de la salud; aunque existe consenso en establecer las edades extremas de la fertilidad (menor de 18 y mayor de 35 años) en las mujeres consideradas en riesgo debido a las comorbilidades asociadas $(13,14)$.

No obstante, la federación internacional de ginecología y obstetricia define la maternidad avanzada como la gestación que ocurre en mujeres con edad igual o superior a 35 años (14).

El 10\% de las gestantes encuestadas clasifican en la definición de maternidad avanzada, lo cual es con- 
sistente con algunos estudios de prevalencia de esta condición (14). Sin embargo, la detección de una paciente de 40 años quien ha tenido además del actual, 3 embarazos, de los cuales tuvo un aborto y dos hijos nacidos vivos, el último de los cuales nació hace 15 años reviste una particular importancia por el riesgo tanto para la madre como para el feto (15-16).

\section{Cumplimiento de controles prenatales}

El control prenatal es un conjunto de acciones encaminadas a la protección, seguimiento y acompańamiento a la mujer durante su periodo de gestación, encaminadas a prevenir complicaciones y prepararla para el parto (17).

El promedio de asistencia a controles prenatales por las gestantes del municipio de Los Palmitos es consistente con la recomendación de la OMS de al menos la realización de cuatro controles prenatales durante el embarazo (18); aunque se identificó el incumplimiento en los controles principalmente en el grupo de gestantes en segundo trimestre de embarazo, se observa un mejoramiento en el cumplimiento en el grupo de gestantes en tercer trimestre y un cumplimiento general importante, si se tiene en cuenta que el municipio reporta en su análisis de la situación de salud un cumplimiento de solo el 77\% en el año 2011 (12).

\section{Índice de automedicación}

La automedicación se ha definido de forma clásica como "el consumo de medicamentos, hierbas y remedios caseros por propia iniciativa o por consejo de otra persona, sin consultar al médico". Esta práctica es un tema controvertido, debido a que existen quienes la rechazan de plano, argumentando los daños que puede generar a la sociedad el uso indiscriminado y sin orientación médica de los medicamentos; y quienes la defienden, aduciendo que es la forma de optimizar y equiparar la atención en salud a toda la población (19).
En general, la automedicación tiene un patrón variable de prevalencia y depende entre otros factores, del tipo de población, del grado de información de la comunidad y de la legislación sanitaria existente. Estudios como el de López (19), sitúa la automedicación en 27,3\% para población general, mientras que Mini (20), para mujeres gestantes la sitúa en $10.5 \%$, lo cual puede ser explicable por la alta vigilancia y la constante información de los profesionales de la salud sobre las mujeres embarazadas.

El índice de automedicación en población general y en mujeres embarazadas es variable (3, 19-21), lo cual puede explicarse por las características socio-demográficas y culturales de la población estudiada. En el caso del municipio de Los Palmitos un índice de automedicación del 21\%, puede considerarse razonable teniendo en cuenta el nivel socio-económico de la población. Sin embargo, en el caso de mujeres embarazadas, debe tenerse en cuenta el factor de riesgo teratogénico para precisar qué tan peligrosa puede ser la automedicación en esta población.

\section{Riesgo de los medicamentos consumidos}

Los medicamentos implican un riesgo en su utilización tanto por la ocurrencia de eventos adversos como por la posibilidad de generar toxicidad para quien los consume, lo anterior se construye en un aspecto altamente importante en el embarazo porque el riesgo no solo es para la madre sino también para el feto. No obstante, las mujeres embarazadas también enferman y en un momento dado se les deben administrar medicamentos, por lo que resulta útil conocer el grado de riesgo que puede representar la administración de un medicamento (21).

De acuerdo a la clasificación del riesgo por exposición a medicamentos de la FDA, las mujeres embarazadas del municipio de Los Palmitos están expuestas a un riesgo considerablemente alto teniendo en cuenta que el $71 \%$ de los medicamentos 
y productos fito terapéuticos que consumieron están ubicados en las categorías C, D y X, las cuales constituyen el mayor riesgo para la gestación (2-3, 7, 20-21).

\section{Plantas medicinales en el embarazo}

Las plantas naturales consumidas durante el embarazo constituyen un riesgo importante para el desarrollo del feto, debido a la presencia de gran cantidad de metabolitos secundarios, que pueden ser farmacológicamente activos y estar asociados a propiedades citotóxicas y mutagénicas que en la mayoría de los casos no se ha llegado a identificar ni cuantificar (22).

Sin embargo, el consumo de plantas naturales en la población general y específicamente en mujeres embarazadas es frecuente en muchos países, incluido Colombia $(22,23)$.

El presente estudio permitió identificar, mediante el ejercicio de la vigilancia del consumo de medicamentos, la utilización de dos plantas naturales con potencial riesgo para el feto. Se trata de las plantas Itamo real (Pedilanthus tithymaloides L. Poit) y Cola de caballo (Equisetum giganteum L.).

Itamo real (Pedilanthus tithymaloides L. Poit) ha sido ampliamente estudiado en el caribe, principalmente en Cuba, de donde proviene su nombre común $(8,24,25)$. Se usa ampliamente en toda la cuenca del caribe como neurosedante, irritante, emético, anti-blenorrágico, anti-herpético, anti-verrugas, contra carcinomas y para el tratamiento de afecciones sifilíticas, bronquitis, laringitis y aftas bucales (8, 24-26). No obstante lo anterior, algunos estudios $(8,24)$ han precisado propiedades abortivas en esta planta, lo que la ubica en la categoría de alto riesgo para el proceso de gestación.

Cola de caballo (Equisetum giganteum L.) es un planta natural comercializada como suplemento nutricional y como condimento. En Colombia el vademécum de plantas medicinales reconoce la es- pecie Equisetum giganteum L. (27), aunque también se ha reportado la especie E. bogotense (28) en Colombia y Perú, país en donde también se comercializan las especies $E$. arvense, $E$. hiemale, y $E$. myriochaetum (29-31).

Aunque la planta tiene usos reportados como diurético, antiinflamatorio, emoliente, astringente, para el lavado de heridas y llagas, para afecciones gastrointestinales y genitourinarias (27-31), no existen estudios ni evidencia suficiente sobre su eficacia ni sobre su seguridad en el embarazo, por tanto es desaconsejable su uso (9).

\section{Agradecimientos}

Los autores agradecen al CCAV Corozal y a la Escuela de Ciencias de la Salud de la Universidad Nacional Abierta y a Distancia; al Centro de Salud y a las mujeres gestantes del municipio de Los Palmitos - Sucre, por todo el apoyo y cooperación recibida para la realización de este trabajo.

El presente trabajo es un producto del proyecto de investigación PS-10-14 "Evaluación del uso de medicamentos y productos fito terapéuticos en mujeres embarazadas del municipio de Los Palmitos - Sucre, como estrategia de prevención de la mortalidad materna y perinatal' aprobado en la convocatoria 004 de 2014 para la financiación de proyectos de investigación como opción de grado de estudiantes en semilleros de investigación reconocidos por la Universidad Nacional Abierta y a Distancia UNAD.

\section{Referencias}

1. Colombia. Instituto Nacional de Salud. Protocolo de vigilancia en salud de la morbilidad materna extrema. Bogotá; 2015. Disponible en: http:/www.ins.gov.co/lineas-de-accion/ Subdireccion-Vigilancia/sivigila/Protocolos\%20SIVIGILA/ PRO\%20Morbilidad\%20Materna\%20Extrema.pdf

2. Baldon Josiane Poli, Correr Cassyano J, Melchiors Ana Carolina, Rossignoli Paula, Fernández-Llimos Fernando, Pontarolo Roberto. Actitudes y conocimientos de los farmacéuticos comunitarios al dispensar medicamentos a embarazadas. 
Pharmacy pract. (Granada Ed. impr.) [Internet]. 2006; 4(1): 38-43. Disponible en: http://scielo.isciii.es/scielo.php?scrip$\mathrm{t}=$ sci_arttext\&pid=S1885-642X2006000100007\&lng=es.

3. Marín Gustavo Horacio, Cañas Martín, Homar Cecilia, Aimetta Corina, Orchuela Jimena. Uso de fármacos durante el período de gestación en embarazadas de Buenos Aires, Argentina. Rev. salud pública [Internet]. 2010; 12(5): 722-731. Disponible en: http://www.scielo.org.co/scielo.php?script=sci_arttext\&pid=S0124-00642010000500003\&lng=en .

4. Colombia. Gobernación de Sucre. Análisis de la situación de salud con el modelo de determinantes sociales en salud 2013. Sincelejo; 2013. Disponible en: http://www.sucre.gov. co/apc-aa-files/61383166366532633430663865366465/ asis-70000-departamento-de-sucre-2013.pdf

5. Colombia. Departamento Nacional de Estadística DANE. Censo general 2005. [Página web]. Disponible en: http:// www.dane.gov.co/censo/files/libroCenso2005nacional.pdf

6. Profamilia. Encuesta Nacional de Demografía y Salud 2010. [Página web]. Disponible en: http://www.profamilia.org.co/ encuestas $/$ Profamilia/Profamilia/index.php?option=com_content $\&$ view $=$ article $\&$ id $=62 \&$ Itemid $=9$

7. Abad Gimeno, FJ et al. Categorías de riesgo de los medicamentos usados en el embarazo. FAP. [Internet]. 2005; 3(2): 49-61. Disponible en: http://www.juntadeandalucia.es/servicioandaluzdesalud/hinmaculada/web/servicios/tcg/documentos/MEDICACION\%20EMBARAZO\%20Y\%20LACTANCIA/ F\%C3\%A1rmacos\%20y\%20Embarazo.pdf

8. Sánchez Govín Ester, Pérez Lamas Ana María, Chávez Figueredo Diosothys, Hechevarría Sosa Isabel. Caracterización farmacognóstica de Pedilanthus tithymaloides L. Poit. Rev Cubana Plant Med [Internet]. 2005; 10(1): Disponible en: http://scielo.sld.cu/scielo.php?script=sci_arttext\&pi$\mathrm{d}=$ S1028-47962005000100004\&lng=es.

9. EE.UU. Biblioteca Nacional de medicina de los Estados Unidos. Cola de caballo. [Página web]. Disponible en: https://www.nlm.nih.gov/medlineplus/spanish/druginfo/natu$\mathrm{ral} / 843 . \mathrm{html}$

10. Salazar Arango, Andrés et al. Consecuencias del embarazo adolescente en el estado civil de la madre joven: estudio piloto en Bogotá, Colombia. Persona y Bioética, [Internet]. 2009 12(2): Disponible en: http://personaybioetica.unisabana.edu. co/index.php/personaybioetica/article/view/968/1309

11. Cortés Alfaro Alba, Chacón O’Farril Damarys, Álvarez García Ana Gloria, Sotonavarro Gómez Yoslan. Maternidad temprana: repercusión en la salud familiar y en la sociedad. Rev Cubana Med Gen Integr [Internet]. 2015 31(3): Disponible en: http://scielo.sld.cu/scielo.php?script=sci_arttext\&pi$\mathrm{d}=$ S0864-21252015000300012\&lng=es

12. Colombia. Alcaldía municipal de Los Palmitos. Plan de Desarrollo del municipio de Los Palmitos. Los Palmitos; 2012. Disponible en: http://cdim.esap.edu.co/BancoMedios/Documentos\%20PDF/lospalmitossucrepd2012-2015.pdf

13. Martínez-Galiano Juan Miguel. La maternidad en madres de 40 ańos. Rev. cub. salud pública [Internet]. 2016 42(3): 451-
458. Disponible en: http://www.scielosp.org/scielo.php?scrip$\mathrm{t}=$ sci_arttext\&pid=S0864-34662016000300012\&lng=en

14. Balestena Sánchez Jorge Manuel, Pereda Serrano Yadilis, Milán Soler José Raúl. La edad materna avanzada como elemento favorecedor de complicaciones obstétricas y del nacimiento. Rev Ciencias Médicas [Internet]. 2015 19(5): Disponible en: http://scielo.sld.cu/scielo.php?script=sci_arttext\&pi$\mathrm{d}=$ S1561-31942015000500004\&lng=es

15. García Alonso Iván, Alemán Mederos María Mislaine. Riesgos del embarazo en la edad avanzada. Rev Cubana Obstet Ginecol [Internet]. 2010; 36(4): 481-489. Disponible en: http://scielo.sld.cu/scielo.php?script=sci_arttext\&pi$\mathrm{d}=$ S0138-600X2010000400002\&lng=es

16. Zárate Cuello Amparo de Jesús, Guerra García Yolanda, Cuesta Joao. Intervenciones disruptivas al comienzo de la vida humana: un debate bioético y del bioderecho sobre la "pena de muerte" prenatal. Nova [Internet]. 2013 July [citado en 2017 mayo 15] ; 11( 20 ): 129-139. Disponible en: http://www.scielo.org.co/scielo.php?script=sci_arttext\&pi$\mathrm{d}=$ S1794-24702013000200013\&lng=en.

17. Hernández-Betancur Ana María, Vásquez-Truisi Martha Lucía. El cuidado de enfermería comprometido: Motor en la satisfacción de la gestante durante el control prenatal. Rev Univ. salud. [Internet]. 2015; 17(1): 80-96. Disponible en: http://www.scielo.org.co/scielo.php?script=sci_arttext\&pi$\mathrm{d}=$ S0124-71072015000100008\&lng=en

18. Atienzo, Erika E et al. Características de la atención prenatal en adolescentes del Perú, comparación con mujeres adultas. Rev Med Hered. [Internet]. 2016; 27(3): Disponible en: http://www.scielo.org.pe/scielo.php?script=sci_arttext\&pi$\mathrm{d}=\mathrm{S} 1018-130 \mathrm{X} 2016000300003 \& \operatorname{lng}=\mathrm{es} \& \mathrm{nrm}=\mathrm{iso}$

19. López José J., Dennis Rodolfo, Moscoso Sonia M. Estudio sobre la Automedicación en una Localidad de Bogotá. Rev. salud pública [Internet]. 2009; 11(3): 432-442. Disponible en: http://www.scielosp.org/scielo.php?script=sci_arttext\&pi$\mathrm{d}=$ S0124-00642009000300012\&lng=en

20. Mini Elsy, Varas Rocio, Vicuña Yuliana, Levano María, Rojas Luis, Medina Julio, Butron Joese, Aranda Renzo, Gutiérrez Erickson. et al. Automedicación en gestantes que acuden al instituto nacional materno perinatal, Perú 2011. Rev. perú. med. exp. salud pública [Internet]. 2012; 29(2), pp. 212-217. Disponible en: http://www.scielo.org.pe/scielo.php?script=sci_arttext\&pid=S1726-46342012000200007\&lng=es\&nr$\mathrm{m}=\mathrm{iso}$

21. Gallego Úbeda M., Delgado Téllez de Cepeda L., Campos Fernández de Sevilla M. ${ }^{a}$ de los A., Lorenzo Pinto A. de, Tutau Gómez F. Actualización del uso de fármacos durante el embarazo: categorías de riesgo. Farm Hosp. [Internet]. 2014; 38(4): 364-378. Disponible en: http://scielo.isciii.es/scielo.php?script $=$ sci_arttext $\&$ pid $=$ S1 130-63432014000400012\&lng=es

22. Macías-Peacok B et al. Plantas medicinales y embarazo Rev Med Inst Mex Seguro Soc. [Internet]. 2009; 47 (3): 331-334. Disponible en: http://www.medigraphic.com/pdfs/imss/im2009/im093q.pdf

23. Pulido Acuña Gloria Paulina, Vásquez Sepúlveda Patricia de 
las Mercedes, Villamizar Gómez Licet. Uso de hierbas medicinales en mujeres gestantes y en lactancia en un hospital universitario de Bogotá (Colombia). Index Enferm. [Internet]. 2012; 21(4): 199-203. Disponible en: http://scielo.isciii.es/scielo. php?script=sci_arttext $\&$ pid $=$ S1 132-12962012000300005\&lng=es

24. Cabrera Suárez Hirán, Núñez Figueredo Yanier. Efecto sedante de extractos de las hojas de Pedilanthus tithymaloides (L.) Poit. Rev Cubana Plant Med. [Internet]. 2008; 13(4): Disponible en: http://scielo.sld.cu/scielo.php?script=sci_arttext\&pi$\mathrm{d}=$ S1028-47962008000400008\&lng=es

25. Carballo Guerra Caridad, Alfaro López Teresita, Rodríguez Ferradá Carlos Alberto, Ramos Gálvez Silvino Raúl, Palazón López Zoe. Desinfección química de Pedilanthus tithymaloides L. Poit. Rev Cubana Plant Med. [Internet]. 2005; 10(2): Disponible en: http://scielo.sld.cu/scielo.php?script=sci_arttext\&pid=S1028-47962005000200012\&lng=es

26. Cabrera Suárez Hirán R., Ruiz Ana K., Reyes, Figueroa María, Cuéllar Cuéllar. Armando, Valdés Yolanda, Pérez Gil Rita M. Evaluación antitumoral y toxicológica de los extractos alcohólicos de Pedilanthus tithymaloides (L.) Poit. Rev haban cienc méd. [Internet]. 2008; 7(3): Disponible en: http://scielo.sld.cu/scielo.php?script=sci_arttext\&pi$\mathrm{d}=$ S1729-519X2008000300017\&lng=es

27. Colombia. Instituto Nacional de Vigilancia de Medicamentos y Alimentos INVIMA. Vademécum colombiano de plantas medicinales. [PDF]. Disponible en: https://www.minsalud. gov.co/sites/rid/1/Vademecum\%20Colombiano\%20de $\% 20$ Plantas\%20Medicinales.PDF

28. Giraldo Quintero Sara Emilia, Bernal Lizarazú María Consuelo, Morales Robayo Adriana, Pardo Lobo Alesdy Zuled, Gamba Molano Leopoldo. Uso tradicional de plantas medicinales en mercados de Bogotá, D.C. Nova. [Internet]. 2015; 13(23): 73-80. Disponible en: http://www.scielo.org.co/scielo.php?script=sci_arttext $\&$ pid $=S 1794-24702015000100007 \& \operatorname{lng}=$ en

29. León, Blanca. La cola de caballo (Equisetum, Equisetaceae) comercializada y exportada del Perú. Lima. Rev. peru biol. [Internet]. 2012; 19(3): Disponible en: http:// www.scielo.org.pe/scielo.php?script=sci_arttext \&pi$\mathrm{d}=$ S1727-99332012000300018\&lng=es\&nrm=iso

30. Campuzano, S., et al. Determinación de la calidad microbiológica y sanitaria de alimentos preparados vendidos en la vía pública de la ciudad de Bogotá DC. 2015; Nova 13(23): 8192.

31. Flórez, R. A. N. Avances y perspectivas en Síndrome de Asperger. 2014; Nova, 12(21). 